\title{
Validação da Escala de Motivação Acadêmica em universitários brasileiros
}

\author{
Tárcia Rita Davoglio a \\ Bettina Steren dos Santos ${ }^{b}$ \\ Carla da Conceição Lettnin c
}

\section{Resumo}

A avaliação dos processos motivacionais relacionados à aprendizagem, permanência e abandono estudantil na Educação Superior tem despertado interesse acadêmico e demandas por instrumentos padronizados. Este estudo objetivou apresentar evidências de validade psicométrica para a Escala de Motivação Acadêmica (EMA) em amostra de 715 estudantes universitários sul-brasileiros. Os resultados corroboram a validade transcultural da escala, apresentando bons índices de consistência interna e análise fatorial confirmatória coerente com o modelo estrutural de sete fatores. Embora sejam sugeridos alguns ajustes semânticos, a versão brasileira da EMA mostrou-se uma medida confiável e válida para avaliar o construto da motivação relacionada à formação universitária, revelando predomínio de motivação autodeterminada nos estudantes pesquisados. Resultados obtidos a partir da EMA podem ser relevantes para o planejamento pedagógico e políticas voltadas à conclusão dos estudos universitários.

Palavras-chave: Educação Superior. Motivação acadêmica. Avaliação. Estudantes.

\section{Introdução}

$\mathrm{Na}$ atualidade, a Educação Superior torna-se, cada vez mais, um fator estratégico e de oportunidade de desenvolvimento e crescimento das nações e dos indivíduos, alinhado com a promoção da democratização do acesso e a redução das

\footnotetext{
a Pontifícia Universidade Católica do Rio Grande do Sul - PUCRS, Programa de Pós-Graduação em Educação/PPGEdu da Escola de Humanidades. Porto Alegre, Rio Grande do Sul, Brasil.

b Pontifícia Universidade Católica do Rio Grande do Sul - PUCRS, Grupo de Pesquisa Processos Motivacionais em Contextos Educativos - PROMOT. Porto Alegre, Rio Grande do Sul, Brasil.

c Universidade Federal do Rio Grande do Sul - UFRGS, Colégio de Aplicação/CAP. Porto Alegre, Rio Grande do Sul, Brasil.
} 
desigualdades sociais. No Brasil, conforme os dados do Censo da Educação Superior 2013 (BRASIL, 2014), houve aumento no número de matrículas em Instituições de Ensino Superior (IES), de 2012 para 2013, de 3,9\% nos cursos presenciais e de $3,6 \%$ nos cursos a distância. Porém, entre 2012 e 2013, constatou-se redução de 5,7\% no número de concluintes, especialmente nos cursos presenciais de IES privadas. Já em IES públicas, embora se observe aumento no número de concluintes nesse período, evidenciou-se quase $50 \%$ de redução no número de concluintes em cursos a distância.

Tal constatação impulsiona a preocupação com a trajetória acadêmica dos estudantes relacionada à permanência e ao abandono dos cursos de graduação escolhidos conforme as produções recentes das Conferencias Latinoamericanas (GARCIA DE ANDOAÍN et al., 2012; 2013), o que, direta ou indiretamente, remete às questões motivacionais. Conhecer e avaliar os fatores associados aos processos motivacionais dos estudantes poderá fornecer caminhos à adequação das novas políticas públicas destinadas à Educação Superior, estendendo ações que fortaleçam o aproveitamento acadêmico e a permanência até a conclusão do curso.

A motivação é um construto complexo, que ao ganhar relevância no ambiente acadêmico e de aprendizagem escolar, demanda pela compreensão dos diferentes elementos que a constituem e a potencializam. Embora se manifeste no comportamento do estudante, a motivação acadêmica envolve não apenas a sua pessoa, mas o sistema educacional como um todo, além da família e do ambiente social, que afeta o estudante em um interjogo de elementos intrapessoais, interpessoais, contextuais e situacionais (VALLERAND et al., 1992).

Nesse sentido, ressalta-se que a literatura carece de uma definição explícita e objetiva para a motivação acadêmica. No entanto, com base na Self-Determination Theory (SDT) (DECY; RYAN, 1985), e considerando que a definição operacional de motivação se relaciona ao porquê do comportamento (VALLERAND et al., 1992), Wilkesmann, Fischer e Virgillito (2012, p. 2) afirmam que "a motivação acadêmica pode ser entendida como a motivação para decidir pelos estudos universitários e para continuar com os estudos universitários", estando fortemente ligada às razões que levam à continuidade dos estudos na Educação Superior.

As pesquisas contemporâneas sobre os processos motivacionais relacionados à educação nos diferentes níveis de ensino têm sido conduzidas cada vez mais se utilizando de instrumentos de avaliação padronizados. Entre estes, destaca-se a Escala de Motivação Acadêmica (VALLERAND et al., 1989), desenvolvida para avaliar a motivação de estudantes do Ensino Superior, fundamentando-se no 
conceito de motivação intrínseca (MI), motivação extrínseca (ME) e amotivação, segundo a Self-Determination Theory, proposta por Deci e Ryan $(1985,2000)$.

Baseando-se na interação entre o ambiente e as necessidades psicológicas básicas e universais de autonomia, competência e afiliação/pertencimento, a SDT postula que a motivação varia de acordo com o tipo de regulação a que está associada. Essas diferentes formas de regulação produzem alterações qualitativas na motivação, as quais se evidenciam no menor ou maior grau de autodeterminação do indivíduo para realizar escolhas e agir, tendo níveis extremos de distinção (DECI; RYAN, 1985, 2000). O nível mais baixo de autodeterminação, denominado amotivação, implica a ausência de percepção pelo indivíduo de associações entre seus interesses e as ações praticadas. Não há uma significação ou sentido pessoal para as ações, as quais assumem um caráter aleatório, alheio às interferências internas ou externas que incidem sobre as escolhas da pessoa.

Em um nível intermediário dessa sequência, encontra-se a motivação extrínseca, na qual as ações são praticadas em função dos resultados almejados ou das recompensas vislumbradas. A motivação extrínseca, contudo, não é unitária, isto é, pode apresentar-se sob quatro diferentes formas, progressivamente mais próximas da autodeterminação: (a) regulação externa, (b) regulação por introjeção, (c) regulação por identificação, (d) regulação integrada (DECI; KOESTNER; RYAN, 2001; DECI; RYAN, 1985). A regulação externa é a que mais caracteriza a motivação extrínseca, ou seja, o sujeito é movido pelo ímpeto de evitar punições ou conseguir recompensas, agindo sob pressões externas ou internas que pouco consideram seus próprios desejos. A regulação externa por introjeção implica certo grau de internalização dos motivos, mas a pessoa ainda age mais por coerção ou pressão do que por vontade própria. Na regulação externa por identificação, a pessoa aceita a importância de certas ações, por se identificarem com o valor da atividade, já havendo certa autonomia em suas decisões. Todavia, na concepção dos autores da SDT, a motivação do tipo extrínseca mais próxima à autodeterminação é a regulada por integração ${ }^{1}$, na qual a escolha da pessoa é relevante para suas decisões e ações, estando integrada ao self, mesmo que não totalmente livre de alguma influência de efeitos de recompensa ou punição provindos do contexto externo.

Por outro lado, a SDT afirma que o nível mais elevado de qualidade autônoma nas ações e escolhas do indivíduo pressupõe a presença de motivação intrínseca. Esta motivação intrínseca no contexto educacional é definida por uma forma de engajamento em ações ou atividades pelo prazer decorrente de aprender, explorar ou

Embora a motivação integrada faça parte do continuum da SDT, não está discriminada como um fator específico no modelo estrutural da EMA desenvolvido por Vallerand et al. (1989; 1992). 
compreender algo novo (VALLERAND et al., 1992). A pessoa sente-se estimulada, aprecia e aproveita a atividade pelo que esta representa, extraindo benefícios na sua realização, independentemente dos resultados concretos que irá obter.

A partir desses pressupostos da SDT para a compreensão dinâmica dos processos motivacionais, Vallerand et al. (1989) desenvolveram uma escala, denominada Escala de Motivação Acadêmica, para avaliar a motivação, desde uma perspectiva qualitativa, isto é, que considere os tipos de motivação que operam na determinação dos comportamentos dos estudantes universitários.

\section{A Escala de Motivação Acadêmica (EMA)}

A versão original da escala foi desenvolvida em língua francesa do Canadá (Échelle de Motivation em Éducation [EME]) por Vallerand et al. (1989), pressupondo a multifatorialidade dos processos motivacionais. É composta por 28 itens e pontuada em uma escala do tipo Likert de sete pontos. Ao ser testada empiricamente pelos autores, em amostras de estudantes universitários do Canadá (estudo 1: $\mathrm{n}=358$; estudo $2: \mathrm{n}=746$ ), a escala confirmou a presença de sete dimensões, alinhadas com os pressupostos da SDT. Estas formam, então, sete subescalas inter-relacionadas, cada uma constituída por quatro itens, com o propósito de mensurar três tipos de MI e três tipos de ME, além da amotivação. A EME foi posteriormente traduzida para o inglês, originando a AMS (Academic Motivation Scale) (VALLERAND et al., 1992), sendo testada em suas propriedades psicométricas em 745 estudantes universitários canadenses, reproduzindo a estrutura fatorial de sete fatores da versão em francês.

Portanto, o modelo fatorial teórico ou estrutural da EME e da AMS apresenta a motivação intrínseca sob a forma das subescalas motivação intrínseca para conhecimento (MIC), motivação intrínseca para realização (MIR) e motivação intrínseca para experimentar estímulos (MIEE). Além da subescala de amotivação (AMO), o modelo contém ainda três outras subescalas que agrupam as diversas formas de motivação extrínseca: por identificação (MEID), por introjeção (MEIN) e por regulação externa (MERE).

Com base nesse modelo estrutural, a EME e a AMS foram utilizadas em diversos estudos visando especialmente investigar suas propriedades psicométricas, já tendo sido, portanto, traduzidas e/ou validadas para diversos países, como se observa no Quadro 1.

Entre esses estudos, encontra-se a versão em espanhol (Escala de Motivación Educativa [EME-E]), obtida a partir da EME, testada em 636 estudantes 
Quadro 1. Pesquisas que evidenciaram propriedades psicométricas das versões da Escala de Motivação Acadêmica em diferentes países.

\begin{tabular}{|c|c|c|c|c|c|c|}
\hline Autores & Ano & Escala & $\begin{array}{c}\text { Amostra/ } \\
\text { Nacionalidade }\end{array}$ & $\begin{array}{c}\text { Consistência } \\
\text { interna dos } \\
\text { fatores }\end{array}$ & $\begin{array}{c}\text { Estabilidade } \\
\text { temporal }\end{array}$ & $\begin{array}{l}\text { Análise } \\
\text { fatorial }\end{array}$ \\
\hline $\begin{array}{l}\text { VALLERAND } \\
\text { et al. }\end{array}$ & 1989 & EME & $\begin{array}{c}746 \text { estudantes } \\
\text { América do } \\
\text { Norte }\end{array}$ & 0,80 & $\begin{array}{c}0,75 \\
(0,69 \text { a } 0,81)\end{array}$ & 7 fatores \\
\hline $\begin{array}{l}\text { VALLERAND } \\
\text { et al. }\end{array}$ & 1992 & AMS & $\begin{array}{c}745 \text { estudantes } \\
\text { América do } \\
\text { Norte }\end{array}$ & 0,62 a 0,82 & 0,71 a 0,83 & 7 fatores \\
\hline $\begin{array}{l}\text { VALLERAND } \\
\text { et al. }\end{array}$ & 1993 & AMS & $\begin{array}{c}217 \text { estudantes } \\
\text { América do } \\
\text { Norte }\end{array}$ & $\begin{array}{c}0,76 \text { a } 0,86 \\
\text { Identificada }=0,60\end{array}$ & Não consta & $\begin{array}{l}\text { Não } \\
\text { consta }\end{array}$ \\
\hline $\begin{array}{l}\text { COKLEY ; } \\
\text { PATEL }\end{array}$ & 2001 & AMS & $\begin{array}{l}263 \text { estudantes } \\
\text { Etnias variadas }\end{array}$ & 0,70 a 0,86 & Não consta & 7 fatores \\
\hline $\begin{array}{l}\text { FAIRCHILD } \\
\text { et al. }\end{array}$ & 2005 & AMS & $\begin{array}{c}1.406 \text { estudantes } \\
\text { América do } \\
\text { Norte }\end{array}$ & 0,77 a 0,90 & 0,69 a 0,87 & 7 fatores \\
\hline $\begin{array}{l}\text { BARKOUKIS } \\
\text { et al. }\end{array}$ & 2008 & AMS & $\begin{array}{l}911 \text { estudantes } \\
\text { Grécia }\end{array}$ & 0,55 a 0,79 & Não consta & 7 fatores \\
\hline $\begin{array}{l}\text { NÚÑ̃EZ } \\
\text { ALONSO; } \\
\text { MARTÍN- } \\
\text { ALBO; } \\
\text { NAVARRO } \\
\text { IZQUIERDO }\end{array}$ & 2005 & EME-E & $\begin{array}{l}636 \text { estudantes } \\
\text { Espanha }\end{array}$ & 0,67 a 0,84 & 0,69 a 0,87 & 7 fatores \\
\hline $\begin{array}{l}\text { NÚÑEZ } \\
\text { ALONSO }\end{array}$ & 2006 & EME-E & $\begin{array}{l}411 \text { estudantes } \\
\text { Paraguai }\end{array}$ & 0,68 a 0,79 & Não consta & 7 fatores \\
\hline SOBRAL & 2003 & EMA & $\begin{array}{l}269 \text { estudantes } \\
\text { Brasil/DF }\end{array}$ & $\begin{array}{c}0,78 \\
(0,66 \text { a } 0,85)\end{array}$ & Não consta & $\begin{array}{l}\text { Não } \\
\text { consta }\end{array}$ \\
\hline $\begin{array}{l}\text { SMITH; DAVY; } \\
\text { ROSENBERG }\end{array}$ & 2010 & AMS & $\begin{array}{l}2.028 \text { estudantes } \\
\text { Estados Unidos }\end{array}$ & 0,79 a 0,86 & Não consta & 7 fatores \\
\hline VIANA & 2012 & EMA & $\begin{array}{c}278 \text { estudantes } \\
\text { Brasil/SP }\end{array}$ & 0,68 a 0,83 & Não consta & 7 fatores \\
\hline
\end{tabular}

EME: Échelle de Motivacion em Éducation; AMS: Academic Motivation Scale; EME-E: Escala de Motivación Educativa; EMA: Escala de Motivação Acadêmica.

Nota: Não foram realizadas análises fatoriais pelo autor.

Fonte: Elaborado pelas autoras a partir da pesquisa (2014).

universitários da Espanha (NÚÑEZ ALONSO; MARTÍN-ALBO; NAVARRO IZQUIERDO, 2005). Esta versão, em língua espanhola, EME-E, foi posteriormente testada no contexto sul-americano, com uma amostra de 411 estudantes universitários do Paraguai (NÚÑ̃Z ALONSO, 2006). Também no contexto sul-americano, a AMS foi traduzida/adaptada para o português do Brasil por 
Sobral (2003), sendo denominada Escala de Motivação Acadêmica (EMA) e testada inicialmente em uma amostra de 269 estudantes de medicina da Região Centro-Oeste do país. No entanto, o estudo de Sobral limitou-se, a priori, aos procedimentos de tradução/adaptação semântica e análises correlacionais.

Posteriormente ao estudo de Sobral, podem-se encontrar estudos psicométricos envolvendo análises fatoriais para a EMA, como o realizado com 278 estudantes brasileiros do curso de Administração (VIANA, 2012). O estudo de Viana (2012), porém, não teve como objetivo principal a validação da EMA. Além disso, embasou-se em amostra de estudantes substancialmente menor do que a do presente estudo, oriunda de um único curso de graduação e de uma IES pública, tornando justificável a demanda por estudos adicionais de validação para a EMA com estudantes brasileiros, explorando IES privadas, de outras regiões do país e incluindo diferentes cursos de graduação, como se propõe o atual estudo.

Por outro lado, é relevante considerar que, a partir da versão original da AMS, traduzida para o Brasil por Sobral (2003), diversas adaptações e/ou modificações foram realizadas por pesquisadores brasileiros na EMA. Essas alterações produziram diferentes versões que excluíram e/ou incluíram itens na escala, modificando-a substancialmente, sob justificativas diversas, porém, continuaram utilizando a denominação de EMA para identificá-las (GUIMARÃES et al., 2010²; JOLY; PRATES, 2011). Com isso, há a possibilidade de ocorrer erros na comparação de resultados estatísticos e psicométricos, tendo em vista a impossibilidade de comparar escalas cujos itens não sejam idênticos. Destaca-se, portanto, que nesse estudo, utilizou-se a versão de Sobral (2003), oriunda da versão em inglês (AMS), e esta traduzida, a partir da versão original em francês (EME), contendo 28 itens, específicos para o contexto da Educação Superior.

Os resultados psicométricos conhecidos da EMA, em suas diversas versões, posicionam o instrumento como bastante relevante no contexto da motivação acadêmica discente. No entanto, estudos de validação de uma medida demandam pela busca substancial e cumulativa de evidências, em diferentes contextos e com amostras diversificadas. Assim, o presente estudo teve por objetivo analisar as propriedades psicométricas da Escala de Motivação Acadêmica, versão em português do Brasil, obtida por meio da tradução da versão original em inglês (AMS), realizada por Sobral (2003), a partir de uma amostra de estudantes universitários da Região Sul do Brasil. Este estudo quantitativo, de delineamento transversal, propôs-se a testar a validade de construto da EMA por meio de

2 GUIMARÃES, S.; BZUNECK, J.; JOLY, M. Escala de avaliação da motivação para universidade (AMU). Londrina: Universidade Estadual de Londrina / Universidade São Francisco. Manuscrito não publicado. 
análises fatorais e análise de consistência interna dos itens e fatores, bem como apresentar a média descritiva das pontuações segundo o gênero dos participantes, a fim de contribuir para o processo de validação de construto. A validação dessa escala permitirá avançar na pesquisa mais ampla que abarca esse estudo ${ }^{3}$, a qual pretende investigar, na perspectiva transcultural, a motivação, o abandono e a permanência estudantil na Educação Superior.

\section{Método}

\subsection{Participantes}

A EMA foi aplicada em 715 estudantes universitários de uma instituição de Ensino Superior privada, localizada na Região Sul do Brasil. Deste total, 43\% eram homens e $57 \%$ mulheres, com idade média de 22 anos ( $\mathrm{DP}=6,56)$. Entre os participantes, $83 \%$ cursavam entre o $3^{\circ}$ e $7^{\circ}$ semestre de sete cursos de graduação, envolvendo as áreas de Exatas, Humanas, Saúde e Licenciaturas.

\subsection{Procedimentos éticos e de coleta de dados}

O projeto cumpriu protocolos éticos, recebendo aprovação da comissão científica, autorização da Pró-Reitoria Acadêmica e dos coordenadores de cursos, além da assinatura do Termo de Consentimento Livre e Esclarecido pelo participante. Este foi convidado a participar, recebendo prévios esclarecimentos e orientações sobre os procedimentos a serem realizados e objetivos da pesquisa. Foi assegurada a participação voluntária na pesquisa, além do anonimato e confidencialidade das informações coletadas, tendo os protocolos de coleta recebido apenas codificação numérica e manuseio pela equipe autorizada/capacitada. A escala foi autopreenchida pelos estudantes que aceitaram participar, em ambiente coletivo, sob supervisão dos pesquisadores, durante uma das aulas indicadas pela coordenação do curso, nas primeiras semanas do $2^{\circ}$ semestre letivo.

\subsection{Procedimentos de análise de dados}

Os dados foram submetidos a análises descritivas, além da verificação da consistência interna dos itens e fatores da EMA (alfa de Crombach) e de análises fatoriais. Inicialmente, foram rodadas análises fatorais exploratórias com o intuito de confirmar a natureza multifatorial da escala. A relevância da realização de

\footnotetext{
A pesquisa na qual o presente estudo se insere está sendo desenvolvida pelo grupo de Pesquisa PROMOT/PUCRS - Processos Motivacionais em Contextos Educativos - cujo projeto é resultante de uma parceria internacional entre instituições de Educação Superior Sul-Americanas. As diversas etapas da sua implementação contam com apoio da Fundação de Amparo à Pesquisa do Estado do Rio Grande do Sul (FAPERGS), Coordenação de Aperfeiçoamento de Pessoal de Nível Superior (CAPES) e Conselho Nacional de Desenvolvimento Científico e Tecnológico (CNPq), agências às quais expressamos nossos agradecimentos.
} 
análises fatoriais exploratórias (AFE) é para que não se subestime o modo como os itens se agrupam espontaneamente, a partir de dada amostra, sendo bastante útil, especialmente quando os modelos confirmatórios ainda são incipientes para uma determinada população, ou diante de possíveis variações culturais (LATTIN; CARROL; GREEN, 2011). Esse modelo exploratório pode oferecer parâmetros relevantes para especificidades não observáveis no modelo estrutural ou teórico utilizado para a análise fatorial confirmatória (AFC). Já as análises fatoriais confirmatórias exploraram o modelo estrutural de sete fatores oriundo das versões originais da EME e AMS, além do modelo exploratório obtido com os dados atuais. Nas análises fatoriais confirmatórias, para medir os índices de qualidade de ajuste absoluto do modelo fatorial, utilizou-se o SRMR (Standardized Root Mean Square Residual) e a RMSEA (Root Mean Square Error of Approximation). Para SRMR e RMSEA, valores próximos ou abaixo de 0,08 e 0,06 , respectivamente, indicam um ajuste satisfatório do modelo. Outros dois índices, de ajuste relativo - TLI (Tucker-Lewis Index) - e de ajuste comparativo - CFI (Comparative Fit Index) -, foram examinados, esperando-se valores de 0,90 a 0,95 como indicativos de ajuste aceitável (BROWN, 2006). Além disso, considerou-se as cargas fatoriais e erros, e as implicações dos itens no modelo teórico. Foram utilizados os softwares estatísticos SPSS (Statistical Package for Social Science) versão 17.0 e Mplus 6.1.

\section{Resultados}

\subsection{Análises Fatoriais Exploratórias}

Inicialmente, a fim de se verificar como o modelo fatorial da EMA se apresenta no contexto brasileiro, tendo em vista a escassez de estudos que confirmem o modelo estrutural conhecido internacionalmente com a versão original da escala em português do Brasil, foram rodadas AFE. A extração dos fatores pelo Método de Componentes Principais e rotação oblíqua, considerando-se autovalores $\geq 1$, resultou em $\mathrm{KMO}=0,91$ e uma matriz de correlação de cinco fatores. O scree plot (Figura 1), também confirma os cinco fatores, os quais explicam $61,8 \%$ da variância.

O modelo fatorial exploratório obtido reproduziu, de modo idêntico, o modelo estrutural para a ME e a amotivação. No entanto, a MI agrupou os três fatores comuns que a representam na EMA, apresentando-se como um único fator, diferentemente do que o modelo fatorial confirmatório determina. Isso significa que as três subdivisões da MI, propostas pela literatura para a EMA, não se reproduziram espontaneamente nesta amostra. Ainda assim, a MI manteve-se composta pelos mesmos itens do modelo de sete fatores, apenas agrupados em um único fator. A Tabela 1 apresenta a matriz fatorial exploratória dos 28 itens da EMA e respectivas cargas fatoriais. 


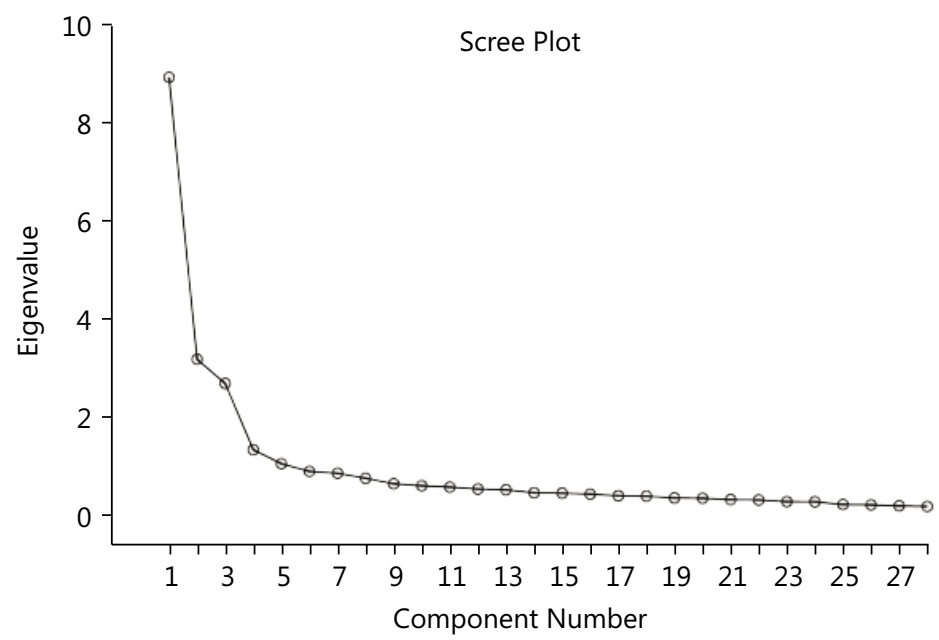

Fonte: Elaborada pelas autoras a partir da pesquisa (2014).

Figura 1. Scree plot resultante da AFE para a amostra de 715 estudantes brasileiros

\subsection{Análises Fatoriais Confirmatórias}

Para a AFC utilizou-se dois modelos estruturais: AFC1 baseada no modelo teórico de sete fatores apresentado pela literatura, composta pelo Fator 1 - Motivação Intrínseca para Conhecimento (MIC; itens 2, 9, 16, 23), Fator 2 - Motivação Intrínseca para Realização (MIR; itens 6, 13, 20, 27), Fator 3 - Motivação Intrínseca para Experiências Estimulantes (MIEE; itens 4, 11, 18, 25), Fator 4 - Motivação Extrínseca por Identificação (MEID; itens 3, 10, 17, 24), Fator 5 - Motivação Extrínseca por Introjeção (MEIN; itens 7, 14, 21, 28), Fator 6 - Motivação Extrínseca por Regulação Externa (MERE itens 1, 8, 15, 22), Fator 7 - Amotivação (AMO; itens 5, 12, 19, 26); AFC2 baseada no modelo de 5 fatores derivado da AFE com a amostra brasileira. Os resultados da AFC com o modelo AFE1, de 7 fatores, que reproduziu o modelo estrutural teórico obtido pelos autores da escala, para os índices de ajuste absoluto foram $\mathrm{RMSEA}=0,07$; $\mathrm{SRMR}=0,06$; e para índices de ajuste incremental foram TLI $=0,92 ; \mathrm{CFI}=0,93$ ), apresentando, assim, adequação da maioria dos índices de qualidade de ajuste recomendados para AFC (RMSEA < 0,08; SRMR < 0,06; TLI e CFI > 0,90). Salienta-se que tais resultados foram obtidos sem introduzir nenhum ajuste ao modelo.

Já o modelo AFE2 evidenciou índices de ajuste próximos aos adequados, sendo $\mathrm{RMSEA}=0,09 ; \mathrm{SRMR}=0,07 ; \mathrm{TLI}=0,90 ; \mathrm{CFI}=0,89$. Mesmo assim, a maioria dos valores estava fora dos parâmetros recomendados pela literatura para o aceite do modelo, confirmando a melhor adequação do modelo teórico de 7 fatores. 
Tabela 1. Análise Fatorial Exploratória da Escala de Motivação Acadêmica na amostra de estudantes brasileiros.

\begin{tabular}{|c|c|c|c|c|c|}
\hline Itens EMA & $1^{\text {a }}$ fator & $2^{a}$ fator & $3^{\circ}$ fator & $4^{\circ}$ fator & $5^{\circ}$ fator \\
\hline Item 9 & 0,91 & & & & \\
\hline Item 2 & 0,89 & & & & \\
\hline Item 16 & 0,77 & & & & \\
\hline Item 25 & 0,75 & & & & \\
\hline Item 11 & 0,70 & & & & \\
\hline Item 20 & 0,70 & & & & \\
\hline Item 18 & 0,69 & & & & \\
\hline Item 23 & 0,66 & & & & \\
\hline Item 4 & 0,59 & & & & \\
\hline Item 6 & 0,57 & & & & \\
\hline Item 13 & 0,51 & & & & \\
\hline Item 27 & 0,47 & & & & \\
\hline Item 28 & & 0,84 & & & \\
\hline Item 7 & & 0,83 & & & \\
\hline Item 21 & & 0,82 & & & \\
\hline Item 14 & & 0,63 & & & \\
\hline Item 22 & & & 0,86 & & \\
\hline Item 15 & & & 0,81 & & \\
\hline Item 8 & & & 0,77 & & \\
\hline Item 1 & & & 0,75 & & \\
\hline Item 24 & & & & 0,81 & \\
\hline Item 3 & & & & 0,80 & \\
\hline Item 17 & & & & 0,72 & \\
\hline Item 10 & & & & 0,66 & \\
\hline Item 26 & & & & & 0,86 \\
\hline Item19 & & & & & 0,75 \\
\hline Item 12 & & & & & 0,73 \\
\hline Item 5 & & & & & 0,73 \\
\hline
\end{tabular}

Nota: A Tabela 1 mostra os 28 itens com cargas fatoriais $>0,35(n=715)$, ordenados pela relevância na extração. $\mathrm{O} 1^{\circ}$ fator correspondeu à motivação intrínseca do modelo teórico da análise fatorial confirmatória norte-americana, porém, os 12 itens que a representam na Escala de Motivação Acadêmica agruparam-se em um único fator $\left(1^{\circ}\right)$; os $2^{\circ}, 3^{\circ}$ e $4^{\circ}$ fatores corresponderam à motivação extrínseca; $05^{\circ}$ fator correspondeu à Amotivação.

Fonte: Elaborada pelas autoras (2014).

Sabe-se que, para a escolha do melhor modelo fatorial, a adequação de todos os índices de ajuste é um critério relevante. Porém, é indispensável que se observe também as cargas fatoriais e erros de medida do modelo (BROWN, 2006), os quais são apresentados nos diagramas de caminho do modelo AFC1 (Figura 2, Figura 3 e Figura 4), corroborando a adequação do modelo. Pode-se observar que as cargas fatoriais dos itens, que se apresentaram positivas, variaram de 0,60 a 0,92. 


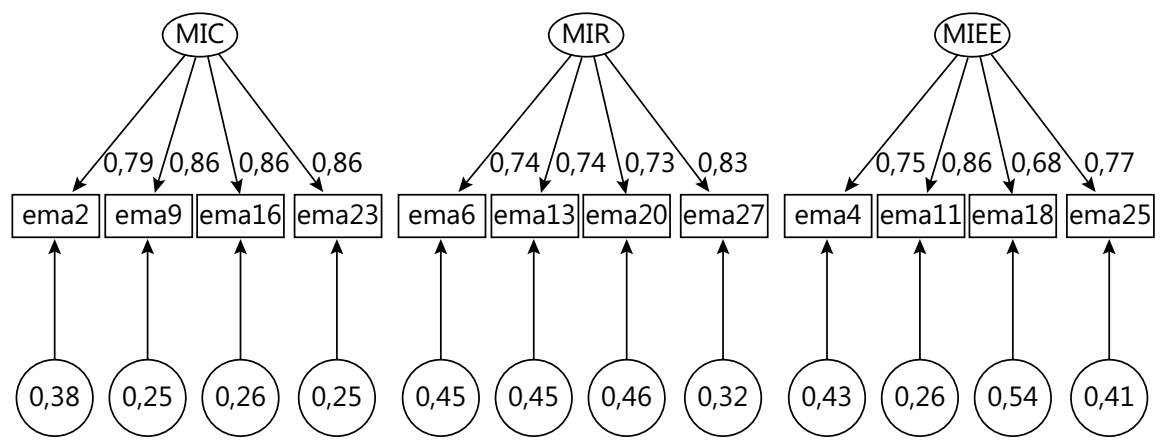

Fonte: Elaborado pelas autoras (2014).

Figura 2. Modelo estrutural para a EMA (AFC1), obtido com AFC para a amostra de estudantes brasileiros com cargas e erros de medida dos itens que compõem os fatores da motivação intrínseca.
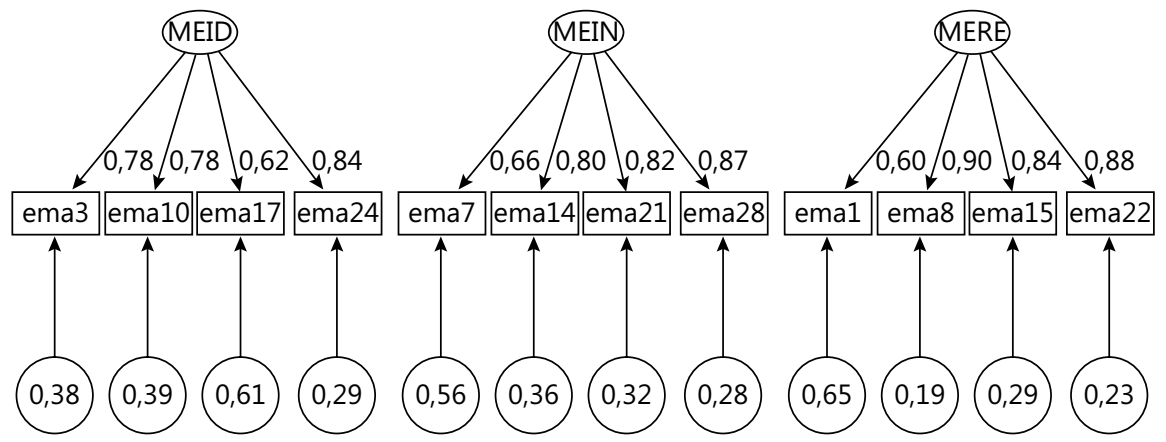

Fonte: Elaborado pelas autoras (2014).

Figura 3. Modelo estrutural para a EMA (AFC1), obtido com AFC para a amostra de estudantes brasileiros, com cargas e erros de medida dos itens fatores que compõem os fatores da motivação extrínseca.

Por outro lado, todos os fatores estão correlacionados entre si, com cargas variando de 0,86 a 0,90 . Como esperado, todos os fatores tiveram correlações negativas com o fator 7 (Amotivação), como mostra a Tabela 2.

\subsection{Confiabilidade dos itens e fatores}

A EMA evidenciou boa consistência interna total para a amostra de 715 estudantes sul-brasileiros $(\alpha=0,89)$. Considerando o modelo estrutural de sete fatores, a consistência interna obtida para os 12 itens que compõe a MI foi $\alpha=0,92$ (MI para conhecimento $\alpha=0,88$; MI para realização $\alpha=0,81$; MI para experiências 
estimulantes $\alpha=0,80$ ); para os 12 itens da ME foi $\alpha=0,84$ (ME por regulação identificada $\alpha=0,77$; ME por regulação introjetada $\alpha=0,83$; ME por regulação externa $\alpha=0,84$ ); e para os 4 itens de amotivação foi $\alpha=0,74$.

Apenas para a ME, observou-se incremento da consistência interna com a exclusão dos itens 1 e 17, elevando os resultados da ME por regulação identificada $(\alpha=0,81)$ e ME por regulação externa $(\alpha=0,85)$.

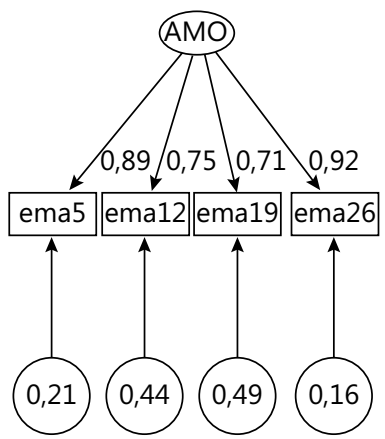

Fonte: Elaborado pelas autoras (2014).

Figura 4. Modelo estrutural teórico para a EMA (AFC1), obtido com AFC para a amostra de estudantes brasileiros, com cargas e erros de medida dos itens que compõem o fator Amotivação.

Tabela 2. Cargas fatorais da Análise Fatorial Confirmatória entre os fatores.

\begin{tabular}{lllllll}
\hline \multirow{2}{*}{ Fatores } & MIR & MIEE & MEID & MEIN & MERE & AMO \\
\cline { 2 - 7 } & \multicolumn{5}{c}{ Cargas Fatoriais } \\
\hline MIC & 0,85 & 0,82 & 0,70 & 0,43 & 0,18 & $-0,56$ \\
MIR & & 0,86 & 0,64 & 0,74 & 0,26 & $-0,46$ \\
MIEE & & 0,58 & 0,49 & 0,09 & $-0,46$ \\
MEID & & & 0,40 & 0,53 & $-0,57$ \\
MEIN & & & & 0,45 & $-0,03$ \\
MERE & & & & & $-0,11$ \\
\hline
\end{tabular}

MIC: motivação intrínseca para conhecimento; MIR: motivação intrínseca para realização; MIEE: motivação intrínseca para experimentar estímulos; MEID: motivação extrínseca por identificação; MEIN: motivação extrínseca por introjeção; MERE: motivação extrínseca por regulação externa; $\mathrm{AMO}$ : subescala de amotivação.

Fonte: Elaborada pelas autoras (2014). 


\subsection{Correlações entre os fatores}

Observou-se, como mostra a Tabela 3 , correlações significativas $(\mathrm{p}<0,01)$ entre a maioria dos fatores. Essas correlações foram positivas e fortes $(\mathrm{r}=0,70)$ entre os três fatores que compõem a motivação intrínseca (MIC, MIR, MIEE) e entre MIR e MEIN $(r=0,59)$; entre MIC e MEID $(r=0,54)$. Correlações positivas e moderadas foram encontradas entre MIEE e MEID $(\mathrm{r}=0,47)$; entre MIR e MEID $(\mathrm{r}=0,46)$; entre MIEE e MEIN $(\mathrm{r}=0,41)$; entre MEID e MERE $(r=0,37)$; entre MIC e MEIN $(r=0,34)$. Correlações positivas e fracas foram encontradas entre MEID e MEIN $(r=0,30)$; entre MIR e MERE $(r=0,16)$; entre MIC e MERE $(r=0,10)$; porém entre MIEE e MERE não houve correlação significativa. Por outro lado, o fator Amotivação evidenciou-se correlações significativas $(\mathrm{p}<0,01)$ e negativas, de fracas a moderadas, com os fatores MIC $(r=-0,39)$; MIR $(r=-0,27)$; MIEE $(r=-0,28)$; MEID ( $\mathrm{r}=-0,37)$. Ressalta-se, também, que não houve correlação entre o Fator 7 (AMO) e os fatores 5 (MEIN) e 6 (MERE).

Adicionalmente, com base nos resultados fatoriais, obteve-se as pontuações médias para os sete fatores da EMA, discriminadas de acordo com o gênero dos estudantes pesquisados. Evidenciou-se, nessa apresentação descritiva (média e desvio-padrão) que a pontuação das estudantes foi sutilmente superior à dos estudantes para os fatores que compõem a motivação intrínseca e a motivação extrínseca. Constatou-se, ainda, que as mulheres obtiveram menores escores no fator Amotivação, como mostra a Tabela 4.

Tabela 3. Correlação entre os sete fatores da EMA na amostra de estudantes sulbrasileiros $(n=715)$.

\begin{tabular}{|c|c|c|c|c|c|c|c|}
\hline Fatores & $\begin{array}{c}\text { Fator } 1 \\
\text { MIC }\end{array}$ & $\begin{array}{c}\text { Fator } 2 \\
\text { MIR }\end{array}$ & $\begin{array}{c}\text { Fator } 3 \\
\text { MIEE }\end{array}$ & $\begin{array}{c}\text { Fator } 4 \\
\text { MEID }\end{array}$ & $\begin{array}{c}\text { Fator } 5 \\
\text { MEIN }\end{array}$ & $\begin{array}{c}\text { Fator } 6 \\
\text { MERE }\end{array}$ & $\begin{array}{c}\text { Fator } 7 \\
\text { AMO }\end{array}$ \\
\hline Fator 1 & & $0,70^{\star \star}$ & $0,70^{\star *}$ & $0,54^{\star *}$ & $0,34^{*}$ & $0,10^{\star *}$ & $-0,39^{* *}$ \\
\hline Fator 2 & & & $0,72^{\star *}$ & $0,46^{\star *}$ & $0,59^{* *}$ & $0,16^{\star \star}$ & $-0,27^{\text {** }}$ \\
\hline Fator 3 & & & & $0,47^{\star *}$ & $0,41^{* *}$ & & $-0,28^{\star *}$ \\
\hline Fator 4 & & & & & $0,30^{* *}$ & $0,37^{\star *}$ & $-0,37^{\star * *}$ \\
\hline Fator 5 & & & & & & $0,34^{* *}$ & \\
\hline
\end{tabular}

MIC: motivação intrínseca para conhecimento; MIR: motivação intrínseca para realização; MIEE: motivação intrínseca para experimentar estímulos; MEID: motivação extrínseca por identificação; MERE: motivação extrínseca por regulação externa; AMO: subescala de amotivação.

Nota ** $p<0,01 ;{ }^{*} p<0,05$.

Fonte: Elaborada pelas autoras (2014). 
Tabela 4. Médias e desvios-padrão dos fatores da Escala de Motivação Acadêmica de acordo com gênero dos estudantes pesquisados.

\begin{tabular}{lccccccc}
\hline \multirow{2}{*}{ Gênero } & \multicolumn{7}{c}{ Fatores da EMA } \\
\cline { 2 - 8 } & MIC & MIR & MIEE & MEID & MEIN & MERE & AMO \\
\hline Masculino & 5,49 & 4,46 & 4,13 & 5,55 & 4,06 & 5,58 & 1,57 \\
(n=308) & $(1,12)$ & $(1,39)$ & $(1,35)$ & $(1,60)$ & $(1,60)$ & $(1,24)$ & $(0,93)$ \\
Feminino & 5,92 & 5,60 & 4,55 & 5,96 & 4,22 & 5,60 & 1,36 \\
$(\mathrm{n}=407)$ & $(1,22)$ & $(1,22)$ & $(1,24)$ & $(0,99)$ & $(1,57)$ & $(1,27)$ & $(0,73)$ \\
\hline
\end{tabular}

EMA: Escala de Motivação Acadêmica; MIC: motivação intrínseca para conhecimento; MIR: motivação intrínseca para realização; MIEE: motivação intrínseca para experimentar estímulos; MEID: motivação extrínseca por identificação; MEIN: motivação extrínseca por introjeção; MERE: motivação extrínseca por regulação externa; AMO: subescala de amotivação. Fonte: Elaborada pelas autoras (2014).

\section{Discussão dos resultados}

A análise fatorial exploratória, cujo objetivo consiste em obter uma matriz fatorial que demonstre as relações espontâneas existentes entre as variáveis observáveis e as dimensões latentes do construto, evidenciou que a estrutura interna da EMA pode ser interpretada a partir de cinco fatores. Deste modo, evidenciou-se que a diferenciação entre MI e ME e a presença de amotivação é possível de ser captada a partir do instrumento. Apesar de a AFE sugerir que os 12 itens que compõe a MI possuem potencial para formarem um único fator, ao invés de três, como estabelece o modelo confirmatório, nenhum dos 28 itens apresentou carga fatorial inferior a 0,40 , variando de 0,47 (item 27) a 0,91 (item 9), apontando que todos podem ser retidos e interpretáveis no modelo.

A análise fatorial confirmatória, por outro lado, apresentou índices de qualidade de ajuste mais satisfatórios quando utilizado o modelo estrutural ou teórico de sete fatores (AFC1), do que o modelo exploratório de cinco fatores (AFC2), este último obtido com a AFE na presente amostra. Ainda que possa ser possível o modelo de cinco fatores, não é, do ponto de vista estatístico, o modelo que melhor se adequa, corroborando o que já havia sido observado na pesquisa empírica com a escala (por exemplo, COKLEY; PATEL, 2001).

Desse modo, todos os índices para o modelo de sete fatores, gerados pelo software Mplus, sem a introdução de nenhum ajuste, estão dentro do limite descrito pela literatura de AFC (LATTIN; CARROL; GREEN, 2011), sendo tão ou mais satisfatórios do que os encontrados nos estudos internacionais que usaram a EME e AMS versões norte-americanas, e a EME-E versão 
espanhola, como mostra a Tabela 5. Pode-se notar, porém, que nos resultados obtidos com outra amostra de estudantes brasileiros (VIANA, 2012) os índices de ajuste incremental foram menos satisfatórios do que os do presente estudo, reafirmando a necessidade de replicar estudos que buscam evidências psicométricas de um instrumento em determinada população para consolidar sua validade.

Com isso, os demais índices que determinam a adequação do modelo estrutural podem ser interpretados, levando a considerar que todas as cargas fatoriais obtidas são superiores a 0,70 , com exceção de 4 itens: $1,7,17,18$. No entanto, mesmo esses itens tiveram cargas acima de 0,60 , o que confirma novamente a validade fatorial do modelo.

Tabela 5. Índices de qualidade do ajuste ${ }^{4}$ de alguns estudos fatoriais da Escala de Motivação Acadêmica.

\begin{tabular}{lccccccc}
\hline Estudo & $\begin{array}{c}\text { Davoglio } \\
\text { et al. }\end{array}$ & $\begin{array}{l}\text { Viana, } \\
\mathbf{2 0 1 2}\end{array}$ & $\begin{array}{c}\text { Núñez } \\
\text { Alonso } \\
\text { et al. } \\
\mathbf{2 0 0 5}\end{array}$ & $\begin{array}{c}\text { Núñez } \\
\text { Alonso } \\
\text { et al, } \\
\mathbf{2 0 0 6}\end{array}$ & $\begin{array}{c}\text { Vallerand } \\
\text { et al., } \\
\mathbf{1 9 8 9}\end{array}$ & $\begin{array}{c}\text { Vallerand } \\
\text { et al., } \\
\mathbf{1 9 9 2}\end{array}$ & $\begin{array}{c}\text { Smith; } \\
\text { Davy; } \\
\text { Rosenberg, } \\
\mathbf{2 0 1 0}\end{array}$ \\
\hline $\begin{array}{l}\text { País } \\
\text { Fatores }\end{array}$ & Brasil & Brasil & Espanha & Paraguai & Canadá & Canadá & EUA \\
No itens & 28 & 7 & 7 & 7 & 7 & 7 & 7 \\
Índices de ajuste absoluto & 28 & 28 & 28 & 28 & 28 & 28 \\
RMSEA & $\mathbf{0 , 0 7}$ & 0,07 & 0,06 & 0,06 & - & - & 0,05 \\
SRMR & $\mathbf{0 , 0 6}$ & - & 0,06 & 0,05 & - & - & - \\
GFI & - & 0,82 & 0,87 & - & 0,92 & 0,94 & 0,94 \\
AGFI & - & 0,77 & & - & 0,91 & 0,91 & - \\
Índices de ajuste incremental/relativo & & & & & \\
CFI & 0,93 & 0,86 & 0,89 & 0,90 & - & - & 0,95 \\
TLI & 0,92 & 0,84 & - & - & - & - & - \\
\hline NFI & - & 0,79 & 0,89 & 0,90 & - & 0,93 & 0,94 \\
\hline
\end{tabular}

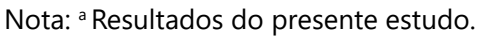

Todos os resultados são sem ajustes a partir de índices de modificação.

RMSEA: Root Mean Square Error of Approximation; SRMR: Standardized Root Mean Square

Residual; GFI: Goodness of Fit Index; AGFI: Adjusted Goodness of Fit Index; CFI: Comparative Fit

Index; TLI: Tucker-Lewis Index; NFI: Normed Fit Index (ajuste bom tem valores GFI > 0,95; AGFI

$>$ 0,9; LATTIN; CARROL; GREEN, 2011).

Fonte: Elaborada pelas autoras (2014).

4 Observam-se variações em alguns tipos de índices apresentados na Tabela 2 em função do software estatístico utilizado para as análises em cada estudo. Estes índices, no entanto, apresentam equivalência na mensuração da qualidade de ajuste. 
Diante das evidências de confiabilidade e validade de conteúdo do instrumento apresentadas neste estudo, pode-se considerar os sete fatores encontrados como sendo dimensões intrínsecas à motivação. Cada fator corresponde a uma dimensão, caracterizadas, de acordo com os autores (VALLERAND et al., 1992, 1993), da seguinte forma:

1) Motivação Intrínseca para conhecimento (MIC): o estudante se engaja em atividades voltadas para a aprendizagem, aprendendo pelo prazer e satisfação decorrentes de explorar ou entender algo novo.

2) Motivação intrínseca para realização (MIR): realizar ou criar algo, superando os limites conhecidos, produz satisfação e prazer, levando o estudante a engajar-se nas atividades.

3) Motivação intrínseca para experiências estimulantes (MIEE): o estudante investe nas atividades, a fim de experimentar as sensações estimulantes e desafiadoras, de natureza sensorial ou estética.

4) Motivação extrínseca por identificação (MEID): o estudante tem um nível razoável de percepção da importância de suas ações e aceitação da responsabilidade própria, envolvendo-se com grau maior de volição e menor sensação de pressão/controle externo.

5) Motivação extrínseca por introjeção (MEIN): baseia-se em contingências externas, sendo controlada por pressões externas, tais como: ofertas implícitas de engrandecimento ou ameaças implícitas de constrangimento. $\mathrm{O}$ estudante age de acordo com certas normas ou expectativas, visando evitar constrangimentos que geram culpa, vergonha ou buscando autoavaliação positiva.

6) Motivação extrínseca por regulação externa (MERE): o estudante sentese pressionado pelos demais a agir de determinada forma. Essa pressão manifesta-se na forma de expectativas de recompensa ou punição concreta ou objetiva.

7) Amotivação (AMO): há interesse ou inspiração, fomentados interna ou externamente, para que o estudante aja em direção a um objetivo acadêmico, manifestando indiferença ou desinteresse.

Por outro lado, a consistência interna total dos itens obtida $(\alpha=0,89)$ é considerada elevada, sendo outro indicador da confiabilidade do instrumento, 
isto é, de que os itens são correlacionados entre si e, portanto, estão medindo algo similar ou complementar. A consistência interna entre os fatores que compõem a MI foi a mais elevada, seguida da ME, reafirmando a coerência entre os itens que compõe cada fator. A consistência interna de cada um dos sete fatores aponta que os alfas encontrados neste estudo são mais elevados dos que os encontrados na maioria dos estudos internacionais, como mostrado no Quadro 1. Por outro lado, nesta amostra, a consistência interna mais baixa foi observada no fator ME por identificação, reproduzindo os achados de outras pesquisas (por exemplo, VALLERAND et al., 1993; VIANA, 2012). A exclusão do item 17, o qual compõe o fator MEID, poderia determinar a elevação do alfa desse fator para 0,81 , o que aponta que este item pode ser responsável pela menor consistência interna observada no fator. Além dele, apenas a exclusão do item 1, que compõe a MERE, produziria aumento no valor de alfa, mas de modo menos significativo, o que reafirma a confiabilidade geral da EMA. Quando comparados os valores de consistência interna da EMA deste estudo com os resultados encontrados em amostras brasileiras (SOBRAL, 2003; VIANA, 2012), nota-se que são muito similares para todos os sete fatores, o que confirma a invariância amostral da confiabilidade da escala.

Ainda em relação ao fator MEID, deve-se salientar que estudos contemporâneos têm discutido a posição deste fator no continuum da motivação autodeterminada (por exemplo, FAIRCHILD et al., 2005; NUÑEZ ALONSO, 2006). Isso porque a sua posição tem se mostrado, de acordo com resultados empíricos, mais próxima da regulação extrínseca do que da motivação intrínseca, ou seja, deveria haver a troca de posição com a regulação introjetada, demandando maiores estudos teóricos sobre a questão. Destaca-se, porém, que nesta amostra sul-brasileira, isso não se confirmou, ou seja, a motivação extrínseca por regulação identificada correlacionou mais forte com dois fatores da motivação intrínseca (MIC e MIEE) do que a motivação por regulação introjetada, com exceção para o fator motivação intrínseca para realização (MIR).

De modo geral, constatou-se que há equilíbrio na contribuição dos seis fatores implicados na composição da motivação dos estudantes que participaram deste estudo em relação à formação superior, apontando para a relevância de se considerar tanto os elementos intrínsecos quanto os extrínsecos nas políticas envolvidas na permanência dos estudantes.

As médias paras os escores fatoriais da EMA na amostra de estudantes deste estudo, por gênero, reproduziram os resultados descritivos muito semelhantes aos encontrados em outro estudo brasileiro (VIANA, 2012) e também no estudo 
inicial de Vallerand et al. (1989) com estudantes norte-americanos. Levando-se em conta apenas médias, evidenciaram-se melhores níveis motivacionais nas mulheres, em praticamente todos os fatores, como mostra a Tabela 6 .

Por outro lado, as mulheres brasileiras também evidenciaram menor pontuação média no fator amotivação do que os homens, o que já havia sido constatado por Viana (2012), porém evidenciando menores discrepâncias entre os gêneros. No entanto, vale a pena destacar que no presente estudo as três maiores médias descritivas entre os escores dos fatores para os homens foi na MERE $(M=5,58)$, MEID $(M=5,55)$ e MIC $(M=5,49)$ e para as mulheres, respectivamente, na MEID $(\mathrm{M}=5,96)$, MIC $(\mathrm{M}=5,92)$ e MIR e MERE ( $M=5,60$ em ambos os fatores), observando-se uma inversão na ordem de prioridade motivacional em relação aos fatores da EMA. Ou seja, tais resultados sugerem que para as mulheres a motivação intrínseca parece ter maior peso no envolvimento no Ensino Superior do que para os homens, embora as diferenças sejam bastante sutis. A comparação estatística da significância das médias poderá precisar a relevância dessas diferenças, pois, outros estudos com amostras internacionais apontam não haver significância estatística nessas diferenças (por exemplo, FAIRCHILD et al., 2005; NÚÑEZ ALONSO; MARTÍN-ALBO; NAVARRO IZQUIERDO, 2005).

Tabela 6. Comparação das médias dos escores fatoriais da Escala de Motivação Acadêmica por gênero entre estudos brasileiros e o estudo original norte-americano.

\begin{tabular}{|c|c|c|c|c|c|c|}
\hline \multirow[b]{2}{*}{ Fatores } & \multicolumn{2}{|c|}{ Estudo atual } & \multicolumn{2}{|c|}{ Viana, 2012} & \multicolumn{2}{|c|}{ Vallerand et al., $1989^{\circ}$} \\
\hline & $\begin{array}{c}M \\
(n=308)\end{array}$ & $\begin{array}{c}F \\
(n=407)\end{array}$ & $\begin{array}{c}M \\
(n=173)\end{array}$ & $\begin{array}{c}F \\
(n=105)\end{array}$ & $\begin{array}{c}M \\
(n=288)\end{array}$ & $\begin{array}{c}F \\
(n=405)\end{array}$ \\
\hline $\begin{array}{l}\text { MI para o } \\
\text { conhecimento }\end{array}$ & $5,49(1,12)$ & $5,92(1,22)$ & $5,31(1,07)$ & $5,32(0,89)$ & 4,87 & 5,17 \\
\hline MI para realizar & $4,46(1,39)$ & $5,60(1,22)$ & $4,41(1,17)$ & $4,55(1,23)$ & 4,29 & 4,45 \\
\hline $\begin{array}{l}\text { MI para } \\
\text { experienciar } \\
\text { estímulos }\end{array}$ & $4,13(1,35)$ & $4,55(1,24)$ & $4,42(1,15)$ & $4,35(1,12)$ & 2,90 & 3,19 \\
\hline ME identificação & $5,55(1,60)$ & $5,96(0,99)$ & $5,78(0,81)$ & $5,88(0,98)$ & 5,62 & 5,89 \\
\hline ME introjetada & $4,06(1,60)$ & $4,22(1,57)$ & $3,84(1,33)$ & $4,16(1,44)$ & 4,18 & 4,67 \\
\hline $\begin{array}{l}\text { ME por regulação } \\
\text { externa }\end{array}$ & $5,58(1,24)$ & $5,60(1,27)$ & $5,71(1,05)$ & $5,91(0,92)$ & 5,09 & 4.98 \\
\hline Amotivação & $1,57(0,93)$ & $1,36(0,73)$ & $2,08(1,10)$ & $1,08(0,91)$ & 1,58 & 1,37 \\
\hline
\end{tabular}

Nota: a ${ }^{O}$ estudo de Vallerand et al. (1989) não apresenta os desvios-padrão.

MI: motivação intrínseca; ME: motivação extrínseca.

Fonte: Elaborada pelas autoras (2014). 


\section{Considerações finais}

No contexto educacional, os processos motivacionais quase sempre são compreendidos em função do grau em que os estudantes investem energia, interesse e esforços nas diversas atividades que compõem os processos de aprendizagem, podendo ou não vir ao encontro das demandas acadêmicas e dos desejos e metas pessoais dos estudantes. Essa motivação direcionada para a aprendizagem e para a permanência no ambiente escolar/acadêmico ainda é muito pouco conhecida e pesquisada nas suas potencialidades para favorecer os resultados acadêmicos exitosos. Entende-se, no entanto, que tais processos motivacionais deveriam ser melhor explorados no planejamento pedagógico, tanto institucional quanto no âmbito de atuação de cada docente. Políticas locais implementadas a partir de dados dessa natureza representam possibilidades de fortalecimento de políticas nacionais para a conclusão dos estudos universitários, reduzindo a discrepância hoje observada entre ingressantes e concluintes, especialmente nos cursos de graduação.

Retomar a versão original da EMA, tal como elaborada pelos autores norte-americanos e utilizada em diversos estudos transculturais, representa tentativa de unificação e padronização da pesquisa empírica. Essa é uma condição fundamental para a viabilidade de estudos comparativos em diferentes culturas e avanços conceituais e teóricos a partir do conhecimento empírico acumulado envolvendo o construto em contextos educativos. No entanto, embora este estudo não tenha se mantido focado sobre questões de tradução/adaptação, observou-se que alguns aspectos semânticos da versão da EMA, traduzida por Sobral (2003), poderiam ser revistos, seguindo critérios explicitamente recomendados pela literatura especializada (BORSA; DAMASIO; BANDEIRA, 2012; PASQUALI, 2010), a fim de tornar os itens ainda mais precisos em decorrência de especificidades transculturais. Como exemplo, no item 25, na versão original em inglês a expressão "high'feeling" (colocada entre aspas pelos próprios autores), na versão espanhola (NÚÑEZ ALONSO; MARTÍN-ALBO; NAVARRO IZQUIERDO, 2005) traduzida como "placer", foi traduzida por Sobral como "euforia", o que, no contexto brasileiro, pode remeter à ideia de excesso, de um tom acima do adequado. Parece oportuna, assim, a sugestão de que, em estudo futuro, a tradução/adaptação da EMA para o português passe por uma revisão seguindo procedimentos metodológicos recomendados pela literatura especializada, como, por exemplo, a tradução reversa (back-translation), visando suprir qualquer limitação semântica.

Por outro lado, os resultados obtidos com a amostra de estudantes sul-brasileiros evidenciaram que a EMA versão brasileira, em sua composição idêntica à original 
produzida pelos autores norte-americanos, contendo 28 itens, é uma medida confiável e psicometricamente válida para mensurar o construto da motivação direcionada para a formação superior. A motivação é aqui concebida como um construto multifatorial, que se compõe de elementos intrínsecos ao self e de outros que se associam a elementos ambientais, internalizados em proporções gradualmente distintas. Além disso, pressupõe também que muitas situações podem se manter indiferentes ao sujeito, não mobilizando elementos que incidem sobre o self, o que leva a um grau de amotivação, ou seja, de ausência de motivos para a significação de uma ação, do ponto de vista interno. Nesse sentido, o presente estudo, com base nas médias descritivas dos fatores da EMA revelou que nesta amostra de uma IES sul-brasileira privada, os estudantes, tantos masculinos quanto femininos, apresentam índices baixos de amotivação se comparados com as médias descritivas para a motivação intrínseca e extrínseca.

É possível, então, inferir que os estudantes pesquisados possuem motivações autodeterminadas em relação às suas perspectivas acadêmicas. As contribuições individuais de cada um dos fatores da EMA nessa constatação devem ser analisadas em maior profundidade e especificidade em estudos futuros com essa população. 


\title{
Validity of academic motivation scale for the Brazilian higher education students
}

\begin{abstract}
Research into the connection between motivational processes to learning and the issue of retention and dropout rates in Higher Education (HE) has been growing steadily due to academic interest and the need for the development of measuring instruments. This study aimed at testing the validity of the Academic Motivation Scale (AMS) using a sample of 715 university students from the South Region of Brazil. Findings have corroborated the transcultural validity of the scale, high internal consistency and confirmatory factor analysis coherent with a seven type structural model. The Brazilian version of AMS seems to be a reliable and valid instrument of measurement to evaluate the issue of motivation in $H E$, revealing that there is a high predominance of self-determination in research students; however, we suggest some minor semantic adjusts. These results could inform educational policies and pedagogical planning aiming at encouraging the successful conclusion of higher education courses.
\end{abstract}

Keywords: Higher Education. Motivation. Students. Evaluation. Academic Motivation.

\section{Validación de la escala de motivación académica en universitarios brasileños}

\section{Resumen}

La evaluación de los procesos motivacionales relacionados al aprendizaje, permanencia y abandono estudiantil en la Educación Superior promueve interés académico y demandas por instrumentos estandarizados. Este estudio objetivo presenta evidencias de validad psicométrica para la Escala de Motivación Académica (EMA) en una amuestra de 715 estudiantes universitarios sur-brasileños. Los resultados corroboran la validad transcultural de la escala, presentando buenos índices de consistencia interna y análisis factorial confirmatoria coherente con el modelo estructural de 7 factores. Aunque sean sugeridos algunos ajustes semánticos, la versión brasileña de EMA se mostró una medida confiable para evaluar el constructo de la motivación universitaria, revelando predominio de motivación auto-determinada en los estudiantes pesquisados. Resultados obtenidos a partir de EMA pueden ser relevantes a la planificación pedagógica y políticas dirigidas a la conclusión de los estudios.

Palabras clave: Educación Superior. Motivación académica. Evaluación. Estudiantes. 


\section{Referências}

BARKOUKIS, V. et al. The assessment of intrinsic and extrinsic motivation and amotivation: validity and reliability of the Greek version of the Academic Motivation Scale. Assessment in Education: Principles, Policy \& Practice, v. 15, n. 1, p. 39-55, 2008. doi:10.1080/09695940701876128

BORSA, J. C.; DAMASIO, B. F.; BANDEIRA, D. R. Adaptação e validação de instrumentos psicológicos entre culturas: algumas considerações. Paidéia (Ribeirão Preto), v. 22, n. 53, p. 423-32, 2012. doi:10.1590/S0103-863X2012000300014

BRASIL. Ministério da Educação. Instituto Nacional de Estudos e Pesquisas Educacionais Anísio Teixeira - INEP. Censo da Educação Superior 2013. Brasília, DF: INEP; 2014. Disponível em: <http://download.inep.gov.br/ educacao_superior/censo_superior/apresentacao/2014/coletiva_censo_ superior_2013.pdf>. Acesso em 9 set 2014.

BROWN, T. E. Confirmatory factor analysis for applied research. New York: Guilford; 2006.

COKLEY, K.; PATEL, N. A psychometric investigation of the Academic Motivation Scale using a United States sample. Measurement and Evaluation in Counseling and Development, v. 34, n. 2, p. 109-19, 2001. doi:10.1177/0013164406288175

DECI, E.; KOESTNER, R.; RYAN, R. M. Extrinsic rewards and intrinsic motivation in education: reconsidered once again. Review of Educational Research, v. 71, n. 1, p. 1-27, 2001. doi:10.3102/00346543071001001

DECI, E.; RYAN, R. M. Intrinsic motivation and self-determination in human behavior. New York: Plenum; 1985.

. The "what" and "why" of goal pursuits: human needs and selfdetermination of behavior. Psychological Inquiry, v. 11, n. 4, p. 227-68, 2000.

FAIRCHILD, J. S. et al. Evaluating new and existing validity evidence for the Academic Motivation Scale. Contemporary Educational Psychology, v. 30, p. 331-58, 2005. doi:10.1016/j.cedpsych.2004.11.001

GARCIA DE ANDOAÍN, J. A. G. et al. Proyecto Alfa: gestión universitária integral del abandono (GUIA). CONFERENCIA LATINOAMERICANA SOBRE EL ABANDONO EN LA EDUCACIÓN SUPERIOR, 2., 2012, Porto Alegre, RS. Madrid: E.U.I.T. de Telecomunicación, 2012. Disponível em: $<$ http://clabes-alfaguia. org/clabes-2012/docs/LibroActas_II-CLABES.pdf>. Acesso em: 10 jun 2014. 
GARCIA DE ANDOAÍN, J. A. G. et al. Proyecto Alfa: GUIA (gestión universitária integral del abandono). In: CONFERENCIA LATINOAMERICANA SOBRE EL ABANDONO EN LA EDUCACIÓN SUPERIOR, 3.. México, DC. Madrid: E.U.I.T. de Telecomunicación, 2013. Disponível em: < http://clabes-alfaguia.org/clabes-2013/docs/Libro_de_ Actas_III_CLABES.pdf $>$. Acesso em: 10 jun 2014.

JOLY, M. C. A.; PRATES, E. R. Avaliação da Escala de Motivação Acadêmica em estudantes paulistas: propriedades psicométricas. Psico-USF, v. 16, n. 2, p. 175-84, 2011. doi:10.1590/S1413-8271201100020000

LATTIN, L.; CARROL, J. D.; GREEN, P. E. Análise de dados multivariados. São Paulo: Cengage Learning; 2011.

NÚÑEZ ALONSO, J. L. et al. Validación de La Escala de Motivación Educativa (EME) en Paraguay. Revista Interamericana de Psicologia, v. 40, n. 3, p. 391-8, 2006.

NÚÑEZ ALONSO, J. L.; MARTÍN-ALBO, L. J.; NAVARRO IZQUIERDO J. Validación de la Version española de la Échelle de Motivation en Éducation. Psicothema, v. 17, n. 2, p. 344-9, 2005.

PASQUALI, L. Instrumentação psicológica: fundamentos e práticas. Porto Alegre: Artmed; 2010.

SMITH, K. J.; DAVY, J. A.; ROSENBERG, D. L. An examination of the validity of the Academic Motivation Scale with a United States business student sample. Psychological Reports, v. 106, n. 2, p. 323-41, 2010. doi:10.2466/pr0.106.2.323-341

SOBRAL, D. T. Motivação do aprendiz de medicina: uso da escala de motivação acadêmica. Psicologia: Teoria e Pesquisa, v. 19, n. 1, p. 25-31, 2003. doi:10.1590/S0102-37722003000100005

VALLERAND, R. J. et al. Construction et validation de l'échelle de motivation en education (EME). Canadian Journal of Behavior Science, v. 21, n. 3, p. 323-49, 1989. doi:10.1037/h0079855

VALLERAND, R. J. et al. The academic motivation scale: a measure of intrinsic, extrinsic, and amotivation in education. Educational and Psychological Measurement, v. 52, n. 4, p. 1003-17, 1992. doi:10.1177/0013164492052004025 
. On the assessment of intrinsic, extrinsic, and amotivation in education: evidence on the concurrent and construct validity of the academic motivation scale. Educational and Psychological Measurement, v. 53, n. 1, p. 159-72, 1993. doi:10.1177/0013164493053001018

VIANA, G. S. Atitude e motivação em relação ao desempenho acadêmico de alunos do curso de graduação em administração em disciplinas de estatística. 2012. 199 f. (Dissertação de Mestrado em Administração) - Faculdade de Economia e Administração, Universidade de São Paulo, São Paulo, 2012.

WILKESMANN, U.; FISCHER, H.; VIRGILLITO, A. Academic motivation of students: the German case. Dortmund: Technische Universität Dortmund; 2012. (Discussion papers, 02-2012). Disponível em: $<$ http://www.zhb.tudortmund.de/wb/Wil/Medienpool/Downloads/DP_2012_21.pdf $>$. Acesso em: 10 jun 2014.

\section{Informações das autoras}

Tárcia Rita Davoglio: Doutorado em Psicologia, Pesquisadora do Programa Nacional de Pós-Doutorado DOCFIX/FAPERGS/CAPES, Professora no Programa de Pós-Graduação em Educação/PPGEdu, da Escola de Humanidades, Pontifícia Universidade Católica do Rio Grande do Sul - PUCRS. Contato: tarcia.davoglio@pucrs.br

Bettina Steren dos Santos: Doutorado em Psicologia Evolutiva e da Educação, Pós-doutora em Educação, Decana Associada e Professora titular da Escola de Humanidades - PUCRS, Pesquisadora Pq2/CNPq, Coordenadora do Grupo de Pesquisa Processos Motivacionais em Contextos Educativos - PROMOT. Contato: bettina@pucrs.br

Carla da Conceição Lettnin: Doutorado em Educação, Professora do Colégio de Aplicação/CAP, da Universidade Federal do Rio Grande do Sul - UFRGS. Contato: carla.lettnin@ufrgs.br 